\title{
Les mélancolies du savoir : la confrontation entre science et réalité dans Manuscrit trouvé à Saragosse de Jean Potocki
}

Nathalie Vuillemin

\section{(2) OpenEdition \\ Journals}

Édition électronique

URL : http://journals.openedition.org/edl/454

DOI : $10.4000 /$ edl. 454

ISSN : 2296-5084

Éditeur

Université de Lausanne

Édition imprimée

Date de publication : 15 décembre 2012

Pagination : 59-78

ISBN : 978-2-940331-29-1

ISSN : 0014-2026

Référence électronique

Nathalie Vuillemin, «Les mélancolies du savoir : la confrontation entre science et réalité dans Manuscrit trouvé à Saragosse de Jean Potocki », Études de lettres [En ligne], 4 | 2012, mis en ligne le 15 décembre 2015, consulté le 20 décembre 2020. URL : http://journals.openedition.org/edl/454 ; DOI : https://doi.org/10.4000/edl.454 


\section{LES MÉLANCOLIES DU SAVOIR: LA CONFRONTATION ENTRE SCIENCE ET RÉALITÉ DANS MANUSCRIT TROUVÉ À SARAGOSSE DE JEAN POTOCKI}

Les principales figures de savant de Manuscrit trouvé à Saragosse s'inscrivent dans une représentation, ancrée depuis l'Antiquité dans la culture européenne, de l'homme de science en grand mélancolique, à la fois susceptible de produire une connaissance particulièrement lucide et pointue du monde, et irrémédiablement séparé de ce dernier. Cet article a pour but de montrer comment, à travers les personnages de Diègue Hervas, Henrique Velasquez et son fils Pedre, Potocki interroge le statut du savoir spécialisé et ses conséquences, aussi bien existentielles qu'épistémologiques et discursives.

Depuis l'Antiquité, l'homme de génie s'est souvent vu attribuer les traits d'un individu mélancolique et solitaire, dont l'enthousiasme créateur ne serait jamais que l'envers d'une profonde inquiétude existentielle. On associe le personnage - poète, philosophe, savant, homme d'Etat - à Saturne, divinité ambiguë, chassée aux confins du ciel pour avoir dévoré ses enfants, représentant ainsi, par son éloignement, l'exil et la tristesse, d'une part, et les plus hautes sphères de la pensée d'autre part ${ }^{1}$. Le sujet né sous le signe de Saturne se situe naturellement "au-dessus» de l'humanité commune et, dans un même mouvement, en marge de celle-ci. Il est plus particulièrement caractérisé par une «irritabilité excessive de la uis imaginatiua" ${ }^{2}$ : tout entier absorbé par la réalisation d'une œuvre extraordinaire, oscillant entre les transports de l'inspiration et la stupeur

I. Pour une analyse détaillée de la signification symbolique de Saturne depuis l'Antiquité, voir R. Klibansky, E. Panofsky, F. Saxl, Saturne et la mélancolie.

2. Ibid., p. 84 . 
qui leur succède, le génie n'est pas au monde. Il est entièrement soumis à l'activité de son esprit.

Le motif est particulièrement cher aux humanistes italiens, qui cultivent l'idéal de l'homo literatus cherchant la réalisation de soi dans un projet parfaitement désintéressé et gratuit: la vie spéculative est un choix, l'affirmation d'une liberté et d'une forme d'indépendance face aux exigences du monde ${ }^{3}$. Mais cette position, à laquelle adhèrent de grandes figures telles que Laurent de Medicis, Policien, ou plus proche de Potocki, Pic de la Mirandole ${ }^{4}$, outre l'isolement auquel elle conduit le savant, en fait le représentant par excellence de la dimension tragique de la destinée humaine: car Saturne, symbole de distance, favorise certes l'émergence de points de vue originaux, de la critique, mais génère également une forme d'hostilité face à la vie; il inspire à la fois l'élan créateur et la pulsion destructrice, le désir d'élévation et le sentiment de la vanité de toute chose 5 .

Les figures de savant de Manuscrit trouvé à Saragosse semblent se placer directement dans la tradition de cette représentation contradictoire de l'activité intellectuelle: Diègue Hervas, Henrique et Pedre Velasquez, pour ne citer que les principaux, sont chacun à leur manière dévoués à leur réflexion jusqu'à l'oubli, parfois, de la réalité concrète. Idéalisée par l'auteur sous certains aspects, parce qu'elle offre la sensation d'une maîtrise extraordinaire (Henrique Velasquez et Diègue Hervas), parce qu'elle conduit à des découvertes fulgurantes et fondamentales (Pedre Velasquez), cette posture est également soumise à une critique ironique, teintée parfois d'amertume: enfermé dans son projet, le savant ne perçoit plus le monde qu'à travers celui-ci. Il développe, en fin de compte, une connaissance paradoxale, incommunicable, dont la validité ne peut qu'être mise en question. La solitude fondamentale du savant potockien traduit ainsi, selon nous, les questionnements de l'auteur lui-même face aux conditions de production et de transmission du savoir.

3. Ibid., p. 393 sq.

4. Pic de la Mirandole est le modèle du grand projet encyclopédique de Diègue Hervas (MTS-1810, p. 493). Comme l'affirment François Rosset et Dominique Triaire, Potocki lui-même peut être rapproché de ces figures de savants universels (F. Rosset, D. Triaire, Jean Potocki, p. 8 sq.).

5. Voir R. Klibansky, E. Panofsky, F. Saxl, Saturne et la mélancolie, p. 400-404. 


\section{De l'isolement salutaire à la perte des références}

Les grandes entreprises intellectuelles que sont l'encyclopédie d'Hervas et le système de Pedre Velasquez ont pour condition originelle une séparation drastique de leur auteur avec le monde: c'est en prison que Don Diègue découvre son omniscience ${ }^{6}$, c'est en prison que le futur géomètre apprivoise sans le savoir la loi du binôme ${ }^{7}$. Métaphore de l'isolement nécessaire du penseur, dont Potocki vante dans ses écrits privés la dimension bienfaisante ${ }^{8}$, la cellule donne lieu à la fois à la révélation de l'être savant, et à la découverte scientifique en tant que telle. Diègue ou Velasquez, en ce sens, incarnent la figure du «savant de laboratoire», dont le statut est controversé au XVIII siècle: certains mettent en doute une connaissance produite dans un espace confiné, théorique, sans lien avec la réalité concrète, lui préférant par exemple l'observation directe dans la nature, ou le voyage, alors que d'autres vantent au contraire l'exactitude et la précision d'expériences effectuées dans des conditions idéales, protégées des aléas du monde?.

Potocki se situe précisément au croisement de ces deux conceptions: grand voyageur, il défend évidemment l'expérience directe du monde, et recommande un exercice scrupuleux de l'observation, qui permettra d'amasser un matériau authentique et indispensable à l'élaboration d'une description précise des phénomènes; mais il se montre également séduit, dans l'analyse des expériences et des documents cumulés, par le raisonnement et son expression mathématique, qui garantirait la production d'un savoir objectif ${ }^{10}$. Potocki, en ce sens, défend la conception galiléenne du savoir, qui refuse le raisonnement pur, tout en niant la validité d'une expérience qui ne recevrait pas un traitement théorique adéquat ${ }^{11}$. On verra par la suite l'importance de cet aspect.

Les épisodes de réclusion de Pedre Velasquez et de Diègue Hervas interrogent tous deux les fondements d'un savoir radicalement séparé

6. MTS-1810, p. 493.

7. MTS-1810, p. 682-685.

8. Voir à ce propos J.-M. Rohrbasser, «Le style géométrique de Potocki (1)», p. 347.

9. Voir à ce propos R. E. Kohler, Landscapes and Labscapes; D. Outram, "New spaces in natural history»; A.-G. Weber, «La littérature et son histoire du point de vue des savants".

Io. Voir D. Triaire, Potocki, chapitre I: «L'épistémologie».

II. Voir E. Namer, "L'intelligibilité mathématique et l'expérience chez Galilée». 
du monde. Le premier, à bien y regarder, découvre bel et bien la loi du binôme sur la base d'une expérience, en utilisant comme support de sa réflexion une fenêtre ${ }^{12}$. Mais l'objet, dont la fonction première est ironiquement d'établir un lien entre l'extérieur et l'intérieur, d'ouvrir sur le monde, est ici dépourvu de toute qualité concrète pour ne plus constituer qu'un ensemble géométrique. L'une des particularités du système de Velasquez serait ainsi de "désémantiser" la réalité, l'apprentissage en réclusion annonçant évidemment les développements ultérieurs du caractère de l'homme: placé dès sa naissance sous le signe de la soustraction, qu'il identifie lui-même comme la base du processus d'abstraction ${ }^{13}$, il n'envisage jamais la réalité que par la combinaison des signes qui la traduisent en langue géométrique, et manifeste en permanence son incapacité à vivre activement dans l'espace concret. On pourra, dès lors, interroger la validité de cette approche purement théorique, qui semble se construire au détriment d'une expérience directe concentrée sur la valeur commune et utilitaire des choses.

Le cas de Diègue Hervas est plus problématique encore. La première idée de l'encyclopédie surgit en effet sur la base d'un simple "appel mental" par lequel Diègue découvre "qu'il avait réellement embrassé tout l'ensemble des connaissances humaines et que, comme Pic de la Mirandole, il eût pu en s'y préparant un peu soutenir des thèses de omni scibili» ${ }^{14}$. Cette apparition fulgurante et pour ainsi dire ex nibilo, dans la logique narrative, d'une science universelle, laisse perplexe: l'encyclopédie, réceptacle de l'ensemble du savoir humain, ne semble plus envisager le monde que comme un référent lointain. Là encore, l'acte de naissance de l'entreprise en annonce le destin malheureux: clos sur lui-même, le grand ouvrage d'Hervas constitue un univers en soi, qui ne semble pouvoir coexister avec la réalité, ou n'entretient avec cette dernière qu'un rapport extrêmement conflictuel: après avoir été dévorée par les rats puis entièrement réécrite, elle accusera un retard tel sur l'état des sciences qu'Hervas sera contraint de consacrer quatre ans à sa correction; enfin terminée, elle sera refusée par le libraire Moreno en raison de sa longueur excessive. Envisagé dès le départ comme un véritable monument

\footnotetext{
I2. $M T S-1810$, p. 682 sq.

13. MTS-1810, p. 707.

I4. MTS-1810, p. 493.
} 
à la gloire de son auteur ${ }^{15}$, l'ouvrage d'Hervas est un objet paradoxal qui n'aura pas d'existence publique. C'est un non-lieu, une pure utopie: la tentative désespérée de fixer les connaissances, de les associer à un nom unique, à une époque où la science se définit sous la double logique dynamique des réseaux et du progrès ${ }^{16}$.

Chez Pedre Velasquez comme chez Diègue Hervas, l'objet d'étude se confond ainsi progressivement avec l'expérience directe de la réalité. Celle-ci n'est jamais perçue que par la médiation d'un savoir qui, dans son ambition d'exhaustivité et d'objectivité, perd progressivement de vue le sens réel des choses. Henrique Velasquez décrit lucidement ce processus, lorsqu'il explique à son fils l'origine de la triste condition des savants comme une forme d'assimilation de soi à la représentation du monde inspirée par la connaissance:

[...] j'enrichis mon intelligence d'une foule de pensées nouvelles; j'en étais rempli, je ne les pouvais contenir. Je sortais pour les révéler aux rochers de Ceuta, je les confiais à la nature, je les offrais en tribut à mon Créateur. Le souvenir de ce que j'avais souffert mêlait à ces sentiments exaltés des soupirs et des larmes qui avaient aussi leurs délices. Elles me rappelaient qu'il était autour de moi des maux que je pouvais adoucir. Je m’unissais en idée aux vues de la providence, aux æuvres de la création, aux progrès de l'esprit humain. Mon esprit, ma personne, ma destinée ne se présentaient point à moi sous une forme individuelle, mais comme faisant partie d'un grand ensemble. Ainsi s'est écoulé l'âge des passions, ensuite j’ai retrouvé le moi ${ }^{17}$.

L'oubli de soi décrit ici par Henrique mêle les principes de la contemplation aux plaisirs de la uita speculatiua chère aux humanistes - recherche tournée, non plus que la contemplation, vers une quelconque forme d'utilité ou de reconnaissance publique, mais dont le sujet se nourrit par amour de la science elle-même ${ }^{18}$. Saturé de ses visions, le savant les projette littéralement sur son environnement ( je ne pouvais

15. MTS-1810, p. 493.

I6. Sur la dimension particulièrement figée de l'encyclopédie d'Hervas, par opposition notamment au projet de Diderot et d'Alembert, voir J.-M. Rohrbasser, «Le style géométrique de Potocki (2)», p. 178.

17. MTS-1810, p. 694. Nous soulignons.

18. MTS-1810, p. 694: "Il faut que je t'apprenne que nous avons aussi nos jouissances; elles sont ignorées et solitaires, mais douces et pures.» 
les contenir [...], je les offrais en tribut à mon Créateur»), tout en se complaisant dans la solitude de cette activité. Ce faisant, comme le suggère Henrique dans la suite du passage, l'âme du savant «repliée en elle-même" l'exclut du monde des hommes et des sentiments ${ }^{19}$. Cette dernière tendance est frappante chez Pedre Velasquez, évidemment, dont Potocki met ironiquement en scène l'incapacité à comprendre les relations humaines autrement qu'à travers des formules mathématiques ${ }^{20}$, mais également chez Diègue Hervas, on y reviendra, que la libido sciendi conduit à négliger toute la dimension sensible de son être.

\section{L'autre monde du savant}

Dans cette configuration, le but initial du savoir, un système idéal en parfaite adéquation avec son objet, qui en dévoilerait le sens en toute transparence, se trouve travesti dans la projection inverse: c'est à la réalité de se conformer aux vues du savant, d'entrer dans le système. La science n'est dès lors plus une simple lecture; c'est l'imposition d'une structure interprétative qui prend valeur de véritable refonte, re-création du réel dans l'espace de la connaissance. Rébecca le laisse entendre lorsqu'elle prie Velasquez d'exposer sa conception de la genèse: "Monsieur le Duc, vous nous aviez promis hier de créer le monde. Dans cette création, vous conformerez-vous à l'œuvre de six jours?» ${ }^{21}$.

La triste fin de Diègue Hervas illustre également ce mouvement. Au comble de sa mélancolie, après avoir abandonné toute ambition quant à la publication de son œuvre, Hervas tombe dans une vision matérialiste de la création qui place à l'origine de toute chose un "acide générateur" et assimile la vie à un processus de fermentation ${ }^{22}$. Il est parallèlement assailli de diverses maladies physiques et psychiques. Son fils, en racontant son histoire, adoptera précisément une rhétorique matérialiste pour décrire cette déchéance: "sa dépouille mortelle était menacée d'une prochaine dissolution " ${ }^{23}$. Le langage est en quelque sorte contaminé par le

19. MTS-1810, p. 694 sq.

20. Dans le rapport qu'il entretient avec sa tante, notamment, dont il ne comprend pas les démarches séductrices (MTS-1810, p. 687-689, puis 700-702).

21. $M T S-1810$, p. 713 . Nous soulignons.

22. MTS-1810, p. 509.

23. MTS-1810, p. 512. 
système qui donne ainsi à un événement commun une forme particulière, reflet d'une interprétation du monde. Dès lors, le savant ne court-il pas le risque d'être pris au piège de la représentation qu'il a construite?

Le personnage d'Henrique Velasquez permet d'aborder la question sous un angle particulièrement intéressant, dans la mesure où il ne partage pas avec son fils Pedre, ni avec Diègue Hervas, le travers d'un intérêt exclusif pour les sciences. Quoique "saisissant tout l'ensemble des connaissances humaines, dans un âge où d'autres à peine en conçoivent les éléments » ${ }^{24}$, Henrique apparait comme un personnage relativement équilibré, intéressé par les affaires publiques, amoureux, altruiste. Son exil à Ceuta est marqué, en premier lieu, par une activité exemplaire d'homme d'Etat ${ }^{25}$. Sa passion pour le progrès des sciences ne l'empêche pas, enfin, de se remémorer sa vie passée, laissant alors libre cours à ses sentiments ${ }^{26}$. Henrique Velasquez, pourtant, est lui aussi pris au piège d'un «système» qui agit, selon nous, comme véritable mise en abyme de la réflexion générale, dans le roman, sur la condition des savants. Il place en effet toute sa vie, à partir de sa disgrâce auprès de Blanche, sous le signe d'un destin funeste: l'incapacité à agir pour son intérêt personnel, tout en favorisant les géniales capacités intellectuelles qu'il partage avec son fils, l'exclurait de l'humanité ${ }^{27}$. Cette conviction semble ancrée de manière précoce dans l'esprit d'Henrique. Après sa malheureuse aventure amoureuse et son consécutif retrait de trois ans dans un couvent, il déclare en effet au ministre qui lui demande des explications quant à la lettre écrite au nom de son frère Carlos: "ce n'était pas cette méprise qui a causé mon malheur. Lors même que le brevet eut été expédié à mon nom, je n'aurais pas été en état d'exercer cette charge [de colonel] ${ }^{28}$. Sibylline, cette déclaration laisse entendre de la part de Velasquez une interprétation bien arrêtée des faits. A partir de là, les différentes étapes de la vie du mathématicien sont-elles effectivement marquées d'une forme de fatalité, ou «choisies» sous l'effet de cette vision?

24. MTS-1810, p. 655 sq.

25. MTS-1810, p. 668 sq. et 676.

26. MTS-1810, p. 672: «[...] souvent le cœur reprenait tous ses droits, ce qui arrivait surtout vers le soir lorsque sa tête s'était épuisée par le travail de la journée.»

27. MTS-1810, p. 693.

28. MTS-1810, p. 667. 
$\mathrm{Au}$ nom de son destin, Velasquez accepte la charge ingrate de commandant à Ceuta, qui implique, comme le souligne le ministre qui la lui propose, une forme de confinement volontaire ${ }^{29}$ :

Je croirai, quittant l'Europe, échapper à la cruelle influence de ma destinée, et qu'en allant dans une autre partie du monde, j'y pourrai trouver le bonheur et la paix sous l'influence d'astres plus favorables ${ }^{30}$.

Evoquant sa vie, Henrique Velasquez se fabrique ainsi un rôle tragique. Les termes qu'il utilise pour expliquer à son fils l'origine de leur étrange condition sont à ce titre significatifs: "bizarrement conformés ${ }^{31}$, les êtres dépourvus d'égoïsme appartiendraient à une "race proscrite»" 32 . On retrouve ici, avec l'abandon d'une terre natale qui participerait d'un malheur atavique, plusieurs motifs attachés au personnage de Phèdre, qui se situe elle aussi dans une généalogie maudite, et attribue aux dieux son amour contre nature pour Hippolyte par cette phrase: "Mon mal vient de plus loin.» ${ }^{33}$. Précisément celle qu'utilise Henrique pour expliquer à son fils l'origine de son malheur:

Tes distractions et mon amour pour Blanche ne sont point les causes premières de nos peines. Notre mal vient de plus loin ${ }^{34}$.

Velasquez serait ainsi poursuivi par Saturne comme Phèdre par Vénus. Sans doute est-ce pour la même raison qu'il remettra son fils à un dieu géomètre, dans le langage qui lui correspond ${ }^{35}$. Potocki, dans ce moment stratégique du texte, joue habilement sur l'association entre cette «fatalité» et la condition de l'homme d'esprit. L'étrange prière baptismale mêle en effet la vision d'une forme d'hérédité dans le malheur aux circonstances très particulières de son histoire:

[...] ô mon Dieu! voilà encore un être sensible que tu as jeté dans l'espace. S'il doit être aussi misérable que l'a été son père, puisse ta

29. MTS-1810, p. 668.

30. MTS-1810, p. 668.

31. MTS-1810, p. 693.

32. MTS-1810, p. 694.

33. Racine, Phèdre, acte I, scène 3, v. 265-278.

34. MTS-1810, p. 693.

35. Saturne est en effet considéré comme la divinité des philosophes, mais aussi, plus spécifiquement, des géomètres. Voir R. Klibansky, E. Panofsky, F. Saxl, Saturne et la mélancolie, p. 230. 
bonté le marquer du signe de la soustraction! [...] Non, mon pauvre enfant, tu ne seras point malheureux comme je l'ai été. Je jure le saint nom de Dieu que jamais je ne t’enseignerai les mathématiques, mais tu sauras la sarabande, le bal de Louis XIV et toutes les impertinences qui parviendront à ma connaissance ${ }^{36}$.

Par un raccourci ironique, les mathématiques sont ici rendues coupables du triste sort de Velasquez, alors que la superficialité de Carlos serait à l'origine de son succès. Raisonnement syllogistique qui, pourtant, conditionnera toute la première éducation de Pedre. Enfermé dans la représentation qu'il s'est faite de son destin, Henrique fabrique littéralement celui-ci, comme Diègue Hervas projette la vision d'un monde matériel impitoyable, et comme Pedre se détache de tout sentiment en vectorisant l'espace des passions.

\section{Le désordre des sentiments dans l'ordre des connaissances}

Dans ces circonstances, toute confrontation avec la réalité est annonciatrice d'une rupture: elle menace en effet la cohérence de l'édifice dans lequel se barricade l'esprit systématique. Le constat est valable pour tous les acteurs de Manuscrit qui, d'une manière ou d'une autre, soumettent le monde à une lecture univoque et en refusent la complexité, l'imprévisibilité. Il n'est que de penser à Alphonse, dont toute l'aventure illustre cette tension entre principes théoriques et expérience concrète. Chez les savants, celle-ci s'avère toutefois beaucoup plus sensible que pour d'autres acteurs du roman, dans la mesure où elle fait vaciller le sens premier d'une entreprise qui, d'une part, se confond avec la vie de son auteur, et d'autre part est sincèrement envisagée comme une explication, un décryptage de la réalité. Absorbé par un projet titanesque ou, dans le cas de Pedre Velasquez, par des réflexions extrêmement exigeantes, le savant perd en outre progressivement tout contact sensoriel et affectif avec son entourage ${ }^{37}$. Le retour soudain de celui-ci dans le

36. MTS-1810, p. 673.

37. Dans la version de 1804, Velasquez expliquait ainsi: «[...] je me rappelle quand il le faut tout ce que j'ai jamais appris sur les sciences exactes, sur l'histoire des hommes et sur celle de la nature, mais d'un autre côté, il m’arrive d'oublier mes rapports momentanés avec les objets qui m'environnent, c'est-à-dire que je ne vois pas ce qu'il y a devant mes yeux et que je n'entends pas ce que l'on me crie aux oreilles.» (MTS-1804, p. 392). 
système en menace alors le fragile équilibre et fait émerger un dangereux conflit entre l'existence du savant et son projet.

Le parcours de Diègue Hervas met en scène à plusieurs reprises ces difficiles «retrouvailles avec le monde». L'épisode de la destruction de l'encyclopédie par les rats, jouant à la fois sur la violence de l'événement et le sentiment d'absurdité qu'il éveille, est évidemment le plus symbolique. Les rats sont en quelque sorte le retour dans l'espace du savant d'une nature que l'aridité de son travail et de son mode de vie avait fait taire. Transformé, en sa présence, en véritable laboratoire de l'abstraction, l'appartement d'Hervas redevient paradoxalement un lieu de vie, appétissant grâce à la colle qui consacre la clôture - impossible - du projet $^{38}$. Avant cette catastrophe, les dangers du repli sur soi s'étaient déjà manifestés en deux autres circonstances: la réception de son premier ouvrage, d'abord, qui signalait une rupture de communication entre le savant et son public. Les Secrets de l'analyse dévoilés conduisent en effet Hervas en prison parce que le titre, en soi, n'est pas accessible dans sa lettre au commun des lecteurs ${ }^{39}$. Par la suite, au moment où s'achève le grand projet de l'encyclopédie - qui permet à Hervas, soit dit en passant, de se remettre très rapidement de ses premiers déboires éditoriaux ${ }^{40}$-, le retour sur les lieux de l'enfance agit à nouveau comme la marque d'une distance un instant comblée entre vie spéculative et vie réelle. Les «larmes de joie dont vingt ans des plus arides conceptions avaient pour ainsi dire tari les sources " ${ }^{41}$ pourraient signifier une forme de réconciliation avec la sensibilité. Mais une fois encore, l'image de l'encyclopédie fait taire les sentiments: «Notre polygraphe eût volontiers passé le reste de ses jours dans sa bourgade native, mais les cent volumes le rappelaient à Madrid.» ${ }^{42}$. Enfin, l'aventure des rats, tout extrême qu'elle apparaisse, est suivie d'un événement qui, décrit très sobrement par le narrateur, n'en semble pas moins représenter le choc le plus violent entre les représentations du savant et la réalité:

38. MTS-1810, p. 504.

39. Diègue Hervas est emprisonné après que des curieux ont interprété le titre Secrets de l'analyse dévoilés comme une critique masquée du gouvernement, et plus particulièrement du ministre des Finances (MTS-1810, p. 492).

40. MTS-1810, p. 494.

4I. $M T S-1810$, p. 503 sq.

42. MTS-1810, p. 504. 
Je vins au monde et ma mère ne survécut que de quelques heures à celle de ma naissance. Hervas n'avait jamais connu l'amour ni l'amitié que par une définition de ces deux sentiments qu'il avait placée dans son soixante et septième volume. La perte de son épouse lui prouva qu'il avait été fait pour sentir l'amour et l'amitié; elle l'accabla plus que la perte de ses cent tomes in-octavo dévorés par les rats ${ }^{43}$.

Hervas, contre toutes les leçons de l'empirisme, n'aurait donc accédé à la connaissance de la vie que par l'intermédiaire du langage, sans que celuici ne repose sur aucune expérience concrète. La souffrance paroxystique que représente la perte de Marica peut dès lors être interprétée comme le constat lamentable d'être passé à côté de la vie réelle. Si le savant se réfugie comme toujours dans le travail, l'enthousiasme qui avait marqué les premières étapes de l'entreprise est remplacé par une mélancolie que renforcera encore sa progressive dégradation physique. A l'instar des rats qui avaient soudain émergé pour anéantir son ouvrage, les maladies et l'hypocondrie qui le rongent de l'intérieur marquent la supériorité de la nature sur l'esprit. Le refus de publier de Moreno achèvera cette progression vers l'anéantissement du projet comme du personnage. A nouveau, l'échec est directement ressenti à travers la comparaison entre la valeur concrète de l'ouvrage et la vie qui lui a été consacrée: "Il voyait sa vie entière perdue, son existence anéantie dans le présent comme dans l'avenir. " ${ }^{44}$. La fin désespérée d'Hervas apparaît alors comme la conséquence directe de ce constat, manifestée comme un soudain besoin, sur le plan métaphysique, de se confronter à l’absurdité de la vie ("Auteur du mal, qui êtes-vous?» ${ }^{45}$ ) et d'y répondre par un ancrage radical dans une conception matérialiste - cyniquement concrète - de l'univers.

\section{Un discours hors du monde?}

Avec l'histoire d'Hervas, Potocki interroge brutalement le sens de l'aventure intellectuelle. Le polymathe, certes, ne partage pas l'optique de désintéressement idéal face aux buts de la science que défend par exemple Henrique Velasquez. Mais même lorsque les savants 
revendiquent les plaisirs que leur procure l'étude en les soustrayant aux vicissitudes du quotidien, force est de constater qu'ils choisissent une activité qui, sous certains aspects, représente un renoncement à la vie réelle. Ainsi Henrique Velasquez "passa-t-il les plus belles années de sa vie à considérer de loin ces grandes batailles où les plus grands génies du monde combattaient avec les armes les plus acérées que l'esprit humain se soit jamais forgées " ${ }^{46}$. Son fils, Pedre, propose dans la version de 1804 une réflexion sur le bonheur qui, certes, peut laisser envisager le calcul comme une réponse positive à l'ennui de l'existence ${ }^{47}$, mais nous oblige, au vu de nos dernières remarques, à interroger le revers de cette représentation:

[la] recherche du bonheur peut, ce me semble, être comparée à la résolution d'une équation d'un degré supérieur. Vous connaissez le dernier terme et vous savez qu'il est le produit de toutes les racines, mais avant d'avoir épuisé les diviseurs, vous arrivez à nombre de racines imaginaires. En attendant la journée se passe et vous avez eu le plaisir de calculer. Il en est de même de la vie humaine: vous y arrivez aussi à des qualités imaginaires que vous avez prises pour des valeurs réelles, mais en attendant vous avez vécu et de plus vous avez agi, or l'action est la loi universelle de la nature ${ }^{48}$.

Qu'est-ce au juste que cette «action" du savant? Et peut-on réellement se satisfaire de la dimension prétendument "gratuite" d'une occupation qui affirme tout de même imposer une forme de savoir, de lecture du monde? ${ }^{49}$ Ces questions peuvent être abordées par l'intermédiaire d'une dernière mise en scène des tensions qui surgissent de la confrontation entre science et réalité, sur le plan des discours cette fois. C'est en effet par le maniement d'un langage que l'homme commun et le savant élaborent et transmettent leur représentation du monde. Celle-ci, nous l'avons dit, repose bien souvent sur des présupposés théoriques ou philosophiques précis, qui en conditionnent la forme; elle est par conséquent toujours porteuse d'une interprétation. Le personnage de Pedre

46. MTS-1810, p. 670.

47. Voir D. Triaire, Potocki, p. 11; J.-M. Rohrbasser, «Le style géométrique de Potocki (1)», p. 323 et 341.

48. MTS-1804, p. 359 sq.

49. Sur l'ambiguïté de l'argument du caractère gratuit de la recherche, voir D. Triaire, Potocki, p. 16-18. 
Velasquez est, dans ce contexte, au centre d'une réflexion intéressante de Potocki sur les conflits auxquels donnent lieu les rencontres des discours et des systèmes qui les conditionnent.

Si l'épisode des rats représente, dans le parcours de Diègue Hervas, l'événement fondateur d'un sentiment d'absurdité face à l'entreprise qu'il s'est assignée, Pedre Velasquez est quant à lui marqué par sa rencontre avec un groupe d'Arabes dont le sheikh lui révèle qu'il est, à leurs yeux, l'incarnation d'un insensé. Velasquez est ainsi confronté à une image de soi incompatible avec la valorisation de l'accumulation des connaissances qui domine la pensée occidentale:

Eh quoi! Me dis-je en moi-même, sur les traces de Locke et de Newton, je serais parvenu aux dernières limites de l'intelligence humaine; appuyant les principes de l'un des calculs de l'autre, j'aurais assuré quelques-uns de mes pas dans les abîmes de la métaphysique, et que m'en revient-il? d'être mis au nombre des fous [...] ${ }^{50}$.

Le jeune homme se voit littéralement aliéné par le miroir déformant que lui tend le sheikh, et comme si les paroles de ce dernier étaient dotées d'un mystérieux pouvoir performatif, devient réellement, pour quelques instants, le fou qu'on vient de lui décrire:

En disant ces mots, je pris mes tablettes et les brisai en petits morceaux [...].

Puis par un mouvement involontaire, je me mis à répéter quelques pas de la sarabande comme faisait mon père lorsqu'il se rappelait ses malheurs.

Les Arabes qui m'avaient vu écrire sur mes tablettes avec beaucoup d'application, ensuite les briser et danser, dirent avec l'accent de la pitié:

- Hamdullah, Allah-kerim! Dieu est grand, louange à Dieu! ${ }^{51}$

Cette scène, dans son caractère excessif, thématise sur le ton de l'ironie le problème de lecture que pose Velasquez à tous les protagonistes du roman. Il est au moment de son apparition un inconnu, incohérent dans ses actions, identifiable par son seul langage à condition, pour le

50. MTS-1810, p. 691. Nous soulignons. Sur la proximité entre les images du savant et du fou, voir D. Triaire, "C'era tre volte un geometra", p. 78.

5I. MTS-1810, p. 691. Nous soulignons. 
spectateur, d'en posséder les fondements. Ainsi hésite-t-on quant à la manière de le présenter: il est «selon monsieur de Penna Velez un géomètre, selon notre aumônier un possédé, et d'après mon faible jugement, [...] un original "52. Plongé dans ses formules, Velasquez ne communique pas; et s'il prend la parole, avant qu'on ne le prie de raconter son histoire, ce n'est que pour donner de la réalité ou des récits qu'il entend une explication qui semble ne s'adresser qu'à lui, et dont le narrateur souligne à l'envi la dimension hermétique: le discours de Velasquez, contrairement aux histoires qui contribuent à réunir la petite société, n'est source que d'apartés; pire, elle conduit souvent à la dissolution du groupe $^{53}$. La conception galiléenne du savoir, qui suppose la maîtrise d'un système de signes très précis pour accéder aux secrets de la nature et, par conséquent, restreint cet accès aux seuls spécialistes ${ }^{54}$, est par là même interrogée. Velasquez, en effet, soumet la nature, mais également la morale, la religion, les sentiments, au filtre des théorèmes et des figures mathématiques. Son principe consiste à soustraire de toute observation particulière les qualités spécifiques ${ }^{55}$ pour tenter une lecture à valeur générale, énoncer une loi, comme le démontre notamment son intervention à l'issue de l'histoire de Torres Rovellas:

- [...] votre histoire m'a vivement intéressé. En vous suivant dans le chemin de la vie [...], j’ai cru voir l'ordonnée d'une courbe fermée s'avancer sur l'axe des abscisses, croître selon une loi donnée, rester presque stationnaire vers le milieu de l'axe, ensuite décroître dans la proportion de son accroissement.

- En vérité, dit le marquis, j'ai bien cru qu’on pouvait tirer quelque morale de mon histoire, mais non pas la mettre en équation.

- Ce n'est pas de votre histoire qu'il s'agit ici, reprit l'inconnu, c'est de la vie humaine en général ${ }^{56}$.

52. MTS-1810, p. 600.

53. Voir MTS-1810, p. 605, 614, 625, 639 et 653.

54. Galilée, L'Essayeur, p. 141: «La philosophie est écrite dans cet immense livre qui se tient toujours ouvert devant nos yeux, je veux dire l'univers, mais on ne peut le comprendre, si l'on ne s'applique d'abord à en comprendre la langue et à connaître les caractères avec lesquels il est écrit. Il est écrit dans la langue mathématique et ses caractères sont des triangles, des cercles et autres figures géométriques, sans le moyen desquelles il est humainement impossible d'en comprendre un mot.»

55. MTS-1810, p. 707.

56. MTS-1810, p. 650. 
D'un point de vue purement logique, la conclusion morale d'une histoire ou la réduction d'un phénomène exemplaire à une équation ne sont pas très différentes: il s'agit d'aller du particulier à l'universel, de faire d'un cas spécifique l'illustration d'une vérité générale. Sur le plan de la communication, en revanche, l'énoncé d'une «morale de l'histoire» se distingue radicalement de la formalisation à laquelle procède Velasquez dans la suite du passage: la première relève en effet d'un usage partagé du langage. Elle a pour vocation d'instruire par le recours à une représentation commune, qui interpelle directement la sensibilité et l'expérience de l'auditeur. Elle prévoit en outre, par la réflexion qu'elle initie, de conduire au bien. Une histoire morale prétend ainsi, d'une certaine manière, exercer une influence sur la réalité, en modifier le cours. L'équation assigne au contraire à la vérité une forme figée: elle prend acte, énonce une régularité immuable qui, rassurante lorsqu'on peut l'appliquer aux lois du mouvement, devient problématique lorsqu'elle prétend prendre en charge l'humain. L'équation des passions, élevée au rang de loi, n'est autre que l'énoncé d'un déterminisme: elle condamne toute histoire future à intégrer un modèle. Sur le plan formel, elle renonce enfin à la dimension émotive du langage qu'exploite au contraire l'histoire à vocation morale et prend le risque d'exclure, le roman l'illustre, quiconque n'est pas familier des signes qu'elle utilise. Lorsque Velasquez prend la parole et lui impose son "style géométrique», l'auditoire, Rébecca mise à part, se tait ${ }^{57}$. Il n'y a pas là communication, mais recherche d'un énoncé définitif, idéalement réductible à un ordre immuable. Le géomètre l'affirme lui-même, l'échange idéal, selon lui, est différé; il renonce à la spontanéité au profit de l'exactitude:

J'ai entendu dire que des joueurs d'échecs avaient fait des parties par lettres, jouant un coup à chaque jour de courrier; voilà proprement comme il faudrait causer et je ne pense pas qu'une repartie faite le même jour puisse avoir quelque justesse ${ }^{58}$.

Se profile alors un paradoxe insoluble: la compréhension du monde, nous l'avons dit, est toujours imposition d'une grille de lecture, traduction et interprétation des données brutes dans le système d'un savoir

57. Sur l'isolement progressif de Velasquez dans les différentes versions du roman, voir D. Triaire, "C'era tre volte un geometra», p. 80 sq.

58. MTS-1810, p. 715. 
donné. La formalisation, aux yeux de Potocki comme pour de nombreux philosophes du XVIII ${ }^{\mathrm{e}}$ siècle, a ceci de séduisant qu'elle renonce à la subjectivité du langage et assure la cohérence logique de l'énoncé59. Mais parallèlement, plus le langage est spécialisé, plus celui qui le manie s'isole, mettant ainsi en péril la possibilité d'une diffusion des connaissances. Velasquez lui-même le suggère à la fin de son exposé, l'expression géométrique a ses limites précisément parce qu'elle renonce, pour assurer une certaine objectivité, à faire image, et par conséquent à signifier:

Lorsque je veux indiquer l'infiniment grand, j'écris un huit couché et divisé par l'unité; lorsque je veux indiquer l'infiniment petit, j'écris l'unité et je la divise par le signe de l'infini; mais ces signes dont je me sers dans le calcul ne me donnent point l'idée de ce que je veux exprimer. [...] J'indique l'infini et je ne le conçois ni ne l'exprime. [...] Ici l'Eglise vient à mon secours: elle me présente l'expression de TROIS contenus dans l'unité sans la détruire. Qu'opposerais-je à ce qui passe ma conception? Je n'ai qu' à me soumettre ${ }^{60}$.

Au contraire du signe géométrique, la Trinité offre à l'esprit le support d'une représentation. L'image permet d'accepter un contenu problématique et impossible à identifier dans la réalité. Mais ce type de représentation est toujours, en réalité, imposition d'un sens: on se soumet, faute de mieux, au discours le plus habile, le plus cohérent en apparence. Comment articuler, dans ces conditions, la confiance en un savoir qui, par accumulation et par association d'idées, permet d'éclairer progressivement des zones d'ombre de la connaissance, à l'inquiétude de ne jamais saisir la réalité qu'à travers le filtre de l'un ou l'autre système? Le savant, en fin de compte, n'affirme-t-il pas ce qu'il veut? ${ }^{61}$ Bien souvent, il est en effet le seul à maîtriser les présupposés de ses analyses. La lecture de la Genèse par Velasquez est ici significative ${ }^{62}$ : elle combine la pratique complexe d'une langue, l'hébreu biblique, à laquelle peu ont accès, à un arrière-plan théorique qui conditionne en partie la traduction proposée

59. Voir D. Triaire, Potocki, chapitre I.

6o. MTS-1810, p. 747. Nous soulignons.

6I. On observera à ce propos la manière dont le système de Diègue Hervas est exposé, Potocki laissant clairement entendre que la production du savoir dépend ici davantage de l'habileté rhétorique du savant que d'une démarche rigoureusement scientifique (MTS-1810, p. 510 sq.).

62. Cette lecture occupe les journées 49 et 50, MTS-1810, p. 713-748. 
par le géomètre. Que signifie un texte voué à révéler une vérité, qui à l'instar du livre de la nature conçu par Galilée, nécessite une connaissance approfondie d'un code et la possession d'un savoir imposant pour être compris?

\section{Conclusion}

La maîtrise de nombreuses sciences, le cumul de connaissances diverses et de points de vue variés se présentent comme l'antidote à cette spécialisation excessive qui contraint la représentation dans une forme figée: il s'agirait de considérer le monde sous toutes ses faces, et non sous l'optique restreinte d'une science, un peu à la manière des naturalistes qui, optant pour une "méthode naturelle», refusent de se limiter à ne considérer que quelques caractéristiques d'un objet pour en épuiser l'ensemble des qualités dans la description. Potocki, tout en construisant des figures de savants qui sombrent sans exception dans la manie du système, souligne à plusieurs reprises, au sein même de leur discours, la tentation de cet idéal véritablement encyclopédique.

Dans cette perspective, le roman agit lui aussi comme une «méthode naturelle" d'appréhension de la réalité: par la multiplication des voix, la confrontation des expériences, des parcours, des regards sur des événements parfois similaires, l'auteur semble chercher à "épuiser le réel" pour en mettre en valeur la richesse. Mais là encore, rien ne garantit d'échapper à la mélancolie qu'insuffle la conscience des limites de toute œuvre; et plus le roman manifeste son ambition d'exhaustivité, plus il tente de refléter la complexité du monde, plus il s'élabore comme un univers à part entière qui, à l'instar de l'encyclopédie d'Hervas, tend à se substituer à la réalité.

Comme nous le rappelle Jean Starobinski, derrière le plaisir gratuit de la lecture, la frénésie narrative que transmettent certains romans, a fortiori lorsqu'ils jouent le jeu de l'enchâssement, se profile peut-être l'inquiétude de voir s'écrouler le monde rassurant de la fiction. Raconter, c'est "peupler le désert", "construire un pont par-dessus de vide» ${ }^{63}$, lutter contre la mélancolie qu'inspire le monde réel, hostile et incompréhensible, pour distendre à l'infini l'espace privilégié que met en place le récit.

63. J. Starobinski, «Prefazione», p. xiii. 
Tous les personnages de Manuscrit trouvé à Saragosse sont, en fait, des «exilés du monde». Et tous savent que celui-ci, jamais, ne sera conforme à l'image qu'en fabriquent leurs histoires.

Nathalie VuiLlemin

Université de Neuchâtel 


\section{BIBLIOGRAPHIE}

\section{Textes}

Galilei, Galileo, L’Essayeur, éd. par Christiane Chauviré, Besançon, Presses Universitaires de Franche-Comté, 1980.

MTS-1804 et MTS-1810 = Ротоскі, Jean, Manuscrit trouvé à Saragosse (version de 1804 et version de 1810), éd. par François Rosset, Dominique Triaire, Paris, Flammarion, 2008 (GF 1342-1343).

\section{Travaux}

Klibansky, Raymond, Panofsky, Erwin, Saxl, Fritz, Saturne et la mélancolie, Paris, Gallimard, 1989.

KoHler, Robert E., Landscapes and Labscapes. Exploring the Lab-Field Border in Biology, Chicago, The University of Chicago Press, 2002.

Namer, Emile, "L'intelligibilité mathématique et l'expérience chez Galilée», in Galilée. Aspects de sa vie et de son ceuvre, Paris, Presses Universitaires de France, 1968, p. 111-126.

Outram, Dorinda, "New spaces in natural history", in Cultures of Natural History, ed. by Nicholas Jardine, John A. Secord, Emma C. Spary, Cambridge, Cambridge University Press, 1996, p. $249-265$.

Rohrbasser, Jean-Marc, "Le style géométrique de Potocki (1)", in Jean Potocki ou le dédale des Lumières, éd. par François Rosset, Dominique Triaire, Montpellier, Presses Universitaires de la Méditerranée, 2010, p. 317-348.

—, "Le style géométrique de Potocki (2)", in Jean Potocki à nouveau, éd. par Emilie Klene, Amsterdam/New York, Rodopi, 2010, p. 131-182. 
Rosset, François, Triaire, Dominique, Jean Potocki. Biographie, Paris, Flammarion, 2004.

Starobinski, Jean, "Prefazione», in Italo Calvino, Romanzi et racconti, I, a cura di Mario Barenghi, Bruno Falcetto, Milano, Mondadori, 1991, p. xi-xxxiii.

Triaire, Dominique, Potocki, Arles, Actes Sud, 1991.

—, "C'era tre volte un geometra", in Archeologia, letteratura, collezionismo, a cura di Elzbieta Jastrzebowska, Monica Niewójt, Roma, Accademia polacca delle scienze, 2008, p. 77-94.

Weber, Anne-Gaëlle, "La littérature et son histoire du point de vue des savants: un dialogue entre Georges Cuvier et Alexander von Humboldt (1800-1845)", Vox poetica, 14/03/2010: http://www. voxpoetica.org/sflgc/biblio/Robineau-Weber.html 